INRA Prod. Anim., 1998, $11(2), 115-124$

\section{R. NOWAK}

URA CNRS / INRA 1291, Laboratoire de Comportement Animal, 37380 Nouzilly

\title{
Développement de la relation mère-jeune chez les Ruminants
}

La mise en place du comportement maternel dépend d'événements physiologiques complexes liés au processus de parturition. L'expression de ce comportement varie quelque peu selon les espèces. Mais ce qui caractérise les ruminants, c'est sans doute la maturité du nouveau-né, extrêmement précoce, et la rapidité avec laquelle un lien d'attachement réciproque s'établit entre la mère et son jeune. C'est à la naissance, en l'espace de quelques heures, que se joue le devenir de la relation mèrejeune. En conditions extensives, le comportement des mères est un facteur important pour la survie du nouveau-né. Un jeune laissé à luimême n'a que peu de chance d'être adopté par une autre femelle. C'est ce lien d'attachement exclusif qui existe entre une mère et son petit qui rend l'adoption d'étrangers (orphelins, abandonnés, surnuméraires) extrêmement difficile.

Chez les mammifères, les interactions mère-jeune sont déterminantes pour la survie du nouveau-né, ne serait-ce que parce que la mère est sa seule source de nourriture. Par conséquent, des soins maternels adaptés aux besoins du nouveau-né représentent un élément essentiel du succès de la reproduction de chaque espèce. En fait le comportement maternel est peut-être plus important pour la reproduction d'un individu que le comportement sexuel puisqu'au cours de son existence, l'échec du cycle parental pénalise le potentiel reproductif de la femelle davantage que l'incapacité temporaire à concevoir. Ceci est particulièrement vrai chez les ruminants pour lesquels la reproduction est saisonnière, la

\section{Résumé}

Chez les ruminants, le comportement maternel présente, immédiatement après la mise-bas, un certain nombre de traits communs comme la consommation des membranes et des liquides fœtaux (les camélidés font exception), l'intérêt pour le nouveau-né et son léchage. Ces soins focalisent l'attention du nouveau-né vers le corps maternel et favorisent le succès des premières prises de colostrum. Après cette phase initiale qui dure quelques heures, les ruminants se subdivisent en deux groupes distincts : les espèces qui cachent leur nouveau-né pendant une durée variable (ex : cerf, chevreuil, caprins, bovins), et ceux dont le petit suit sa mère dès la naissance (ex : renne, ovins). La relation mère-jeune est, par ailleurs, caractérisée par une reconnaissance interindividuelle bien développée, base de la sélectivité maternelle : rapidement, la femelle n'accepte d'allaiter que son nouveau-né. La formation du lien mère-jeune s'effectue généralement sans problème. Toutefois, dans certaines conditions, le comportement maternel peut, par son absence ou sa sélectivité, devenir un facteur limitant la production. gestation longue, la taille de la portée réduite, et le développement du jeune lent. On peut aisément admettre qu'une femelle non fécondée lors d'une saillie a toutes les chances de revenir en chaleur au cours de la même saison de reproduction, offrant une possibilité supplémentaire de devenir gestante et d'assurer une descendance. Par contre, le potentiel reproductif d'une femelle dont la portée ne survit pas est fortement pénalisé puisque celle-ci ne pourra alors être re-fécondée qu'à la saison sexuelle suivante.

Les stratégies comportementales quant à l'élevage du jeune varient selon les espèces. Chez les animaux nidicoles, comme les rongeurs chez qui les nouveau-nés sont souvent nombreux et peu développés, la relation mèrejeune s'articule autour du nid plutôt qu'autour des individus; il n'y a pas de relation préférentielle entre la femelle et ses petits. Par contre, chez les ruminants, le nouveau-né est entièrement développé du point de vue sensori-moteur dès la naissance et rapidement autonome pour sa thermorégulation. La relation mère-jeune se caractérise alors par le maintien d'un contact direct entre la mère et le jeune grâce à une reconnaissance interindividuelle bien développée et l'établissement d'un lien plus ou moins exclusif. L'élément le plus caractéristique de l'attachement est alors sans doute la rapidité avec laquelle il s'établit, que ce soit de la mère envers le jeune, ou bien du jeune envers la mère. 
Les ruminants se subdivisent en plusieurs familles. Celles présentant un intérêt agronomique sont les suivantes : les cervidés (cerf, renne, chevreuil), les bovinés (vache, bison, buffle, yack), les caprinés (chèvre), les ovinés (mouton) et les camélidés (chameau, droma-

Figure 1. Evolution de l'attraction envers le liquide amniotique à l'œstrus et autour de la parturition chez la vache et la brebis. Les animaux sont mis en situation de choix alimentaire où une des deux mangeoires est contaminée avec du liquide amniotique; l'attraction est mesurée par la proportion de femelles s'alimentant plus de 5 secondes dans la mangeoire contaminée (Lévy 1985, Lévy et al 1983).

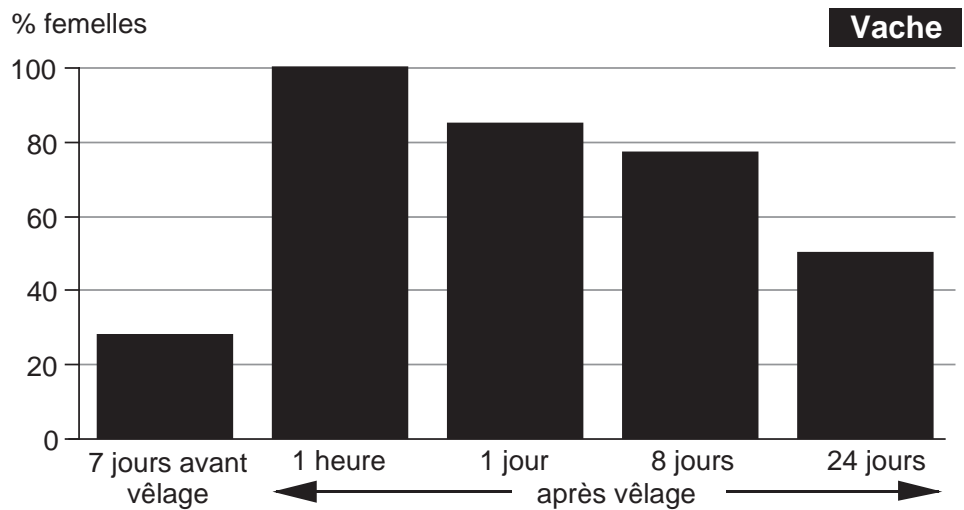

$\%$ femelles
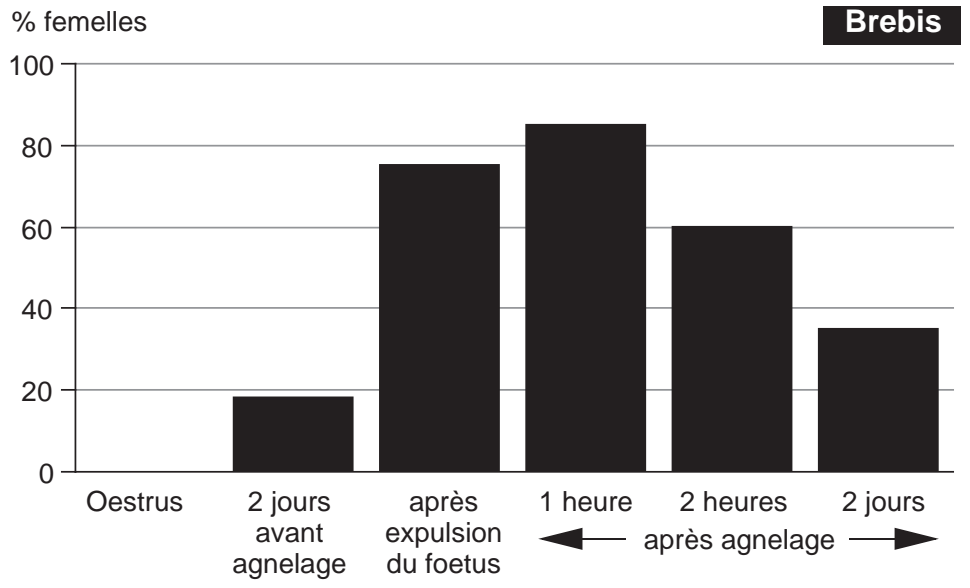

Le léchage du nouveau-né commence dans les minutes qui suivent la mise-bas. Cliché R. Nowak.

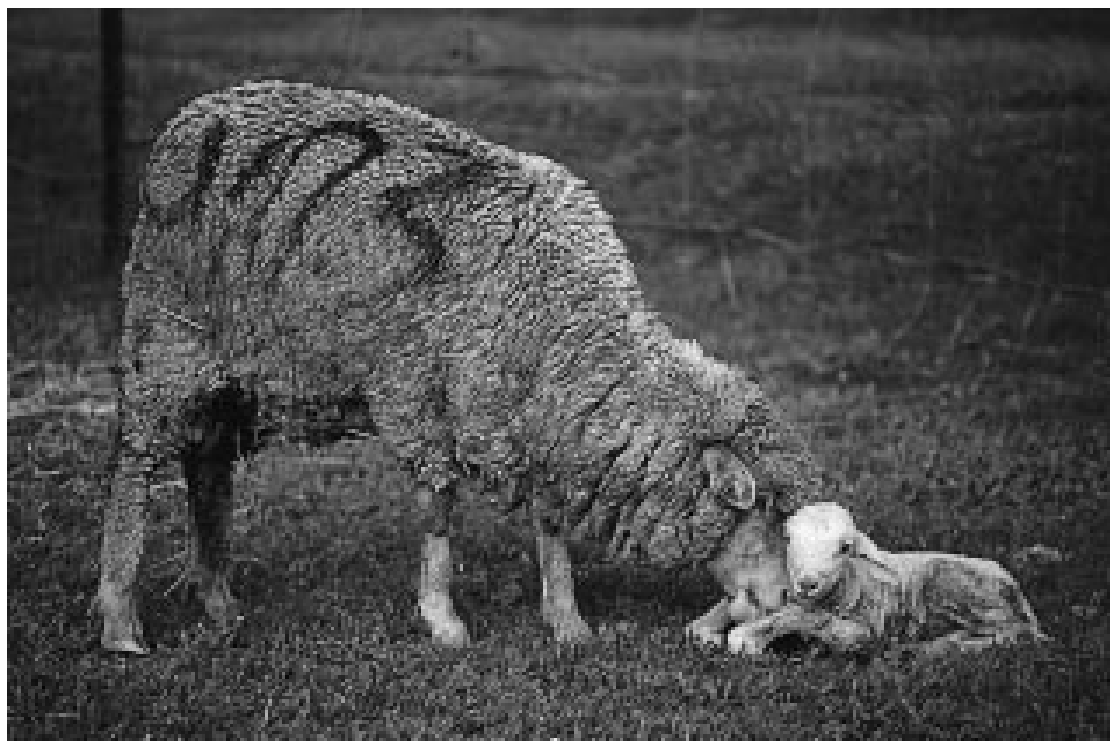

daire, lama). La plupart des connaissances acquises actuellement sur le comportement maternel sont issues d'espèces ayant un impact économique important : ovins, caprins, bovins. C'est pourquoi elles serviront de base pour cette comparaison, même si l'extrapolation de résultats aux espèces sauvages est toujours hasardeuse. Les autres espèces n'ont fait l'objet que de quelques études descriptives car elles constituent pour la plupart un élevage marginal. Néanmoins, les cervidés, ainsi que certains camélidés et bovidés, suscitent un intérêt en France comme en Europe car ils offrent la possibilité d'une diversification en production animale dans un contexte économique de plus en plus difficile, et représentent une solution potentielle dans le cadre d'une politique d'entretien de l'espace (Thimonier et Sempéré 1989, Prud'hon et al 1993, Faye et al 1995, Agabriel et al 1996).

\section{1 / Comportement péripartum}

On distingue deux types de femelles selon le comportement qu'elles adoptent immédiatement après la mise-bas : les femelles actives et les femelles passives (Lent 1974). Les camélidés sont de type passif : les mères ne lèchent pas leur petit ni ne cherchent à le débarrasser de ses membranes placentaires (dromadaire : Gauthier-Pilters et Dagg 1981, lama : Prud'hon et al 1993). Toutefois, elles utilisent leurs lèvres et leur langue pour stimuler la miction et la défécation du nouveauné. Cette particularité comportementale n'a jusqu'alors trouvé aucune explication quant à sa finalité biologique. Chez les femelles actives, qui sont la vaste majorité chez les ruminants, le comportement de léchage du nouveau-né commence dans les minutes qui suivent la mise-bas. Il reflète l'attraction soudaine que développent les femelles périparturientes envers le liquide amniotique. En effet, on a pu montrer chez la brebis, et dans une moindre mesure chez la vache, que ce liquide était naturellement répulsif pendant la quasi totalité de la vie de la femelle. Les changements physiologiques liés à l'expulsion du fœtus le rendent temporairement attractif (Lévy 1985, Lévy et al 1983 ; figure 1). Les observations réalisées chez les ruminants domestiques (bovins, ovins, caprins) et sauvages (cerf, bison) montrent que la femelle dirige son attention d'abord vers la tête du nouveau-né avant de lécher le reste du corps (Lent 1974). Toutefois, lorsque celui-ci cherche à se lever, la mère oriente son activité de léchage vers la région anogénitale ce qui aurait pour effet de stimuler la miction et la défécation au moins chez certaines espèces (Alexander 1988). Diverses fonctions ont été attribuées à cette activité de léchage. Outre le fait que le léchage stimule l'élimination, il aide à sécher le pelage du nouveau-né, favorisant ainsi sa thermorégulation néonatale, optimise les premières interactions mèrejeune permettant ainsi à la mère d'apprendre l'odeur de son jeune et au nouveau-né de diriger son attention vers le corps maternel et, de 
par les stimulations tactiles, possède un effet apaisant sur le nouveau-né. Cependant, le léchage n'est pas l'unique manière de stimuler tactilement le nouveau-né. Chez les bovins, ainsi que chez le bison et le chevreuil, il n'est pas rare de voir la mère pousser le jeune du museau, alors que chez les ovins et chez le cerf, des femelles ont été parfois observées en train de gratter le corps du nouveauné à l'aide d'une de leurs pattes antérieures, ce qui l'incite généralement à se lever (Lent 1974). Chez certaines espèces, la femelle consomme également le placenta. Cela est communément observé chez les cervidés, plus rarement chez les bovins et les ovins, mais est totalement absent chez les camélidés (Alexander 1988). La fonction biologique d'un tel comportement a beaucoup été discutée. Outre un apport nutritif et endocrine qui a été suggéré, la placentophagie est considérée comme un comportement antiprédateur, surtout chez les cervidés qui mettent bas en isolement. La femelle " effacerait " la trace olfactive du lieu de mise-bas et minimiserait ainsi la venue de prédateurs potentiels (Lent 1974).

De par la précocité de son développement, le nouveau-né cherche rapidement à se lever et à téter. Dans la plupart des espèces, il se lève avant l'âge d'une heure et trouve la tétine dans l'heure suivante. Un certain nombre d'informations issues du corps maternel aident le jeune dans sa recherche initiale de la mamelle. Les données obtenues chez les ovins montrent que l'agneau nouveau-né est spontanément attiré vers des stimuli qui caractérisent la glande mammaire : la chaleur, la texture de la peau dépourvue de pilosité, l'odeur de la cire inguinale, l'angle formé entre les pattes et le corps de la brebis (Vince 1993). La posture de la mère aide également au succès des premières tétées. Les chèvres et les brebis multipares arrondissent souvent le dos et écartent leur patte postérieure lorsque le jeune s'approche de la zone mammaire facilitant ainsi l'accès au trayon. La plupart des femelles allaitent en position debout, leur petit se présentant parallèlement à leur corps. Une telle position appelée « parallèle inverse » est de loin la plus commune et focalise l'attention de la mère vers la région anale $\mathrm{du}$ jeune permettant ainsi son identification olfactive (Poindron et Le Neindre 1980). A mesure que le jeune grandit, il n'est pas rare de le voir téter perpendiculairement à la mère ou même par l'arrière. Chez les cervidés, les femelles nourrissent parfois leur petit en position couchée. Cela peut s'observer dès la naissance (cerf : Arman 1974) ou même lorsque le jeune est âgé de plusieurs jours voire plusieurs semaines (renne : Espmark 1971a).

\section{2 / Mécanismes physiologiques de la mise en place du comportement maternel}

L'aptitude à manifester un comportement maternel s'établit autour de la parturition. Son apparition est associée aux facteurs phy-
La plupart des femelles allaitent debout, leur petit se présentant parallèlement à leur corps. Clichés A. Béguey.
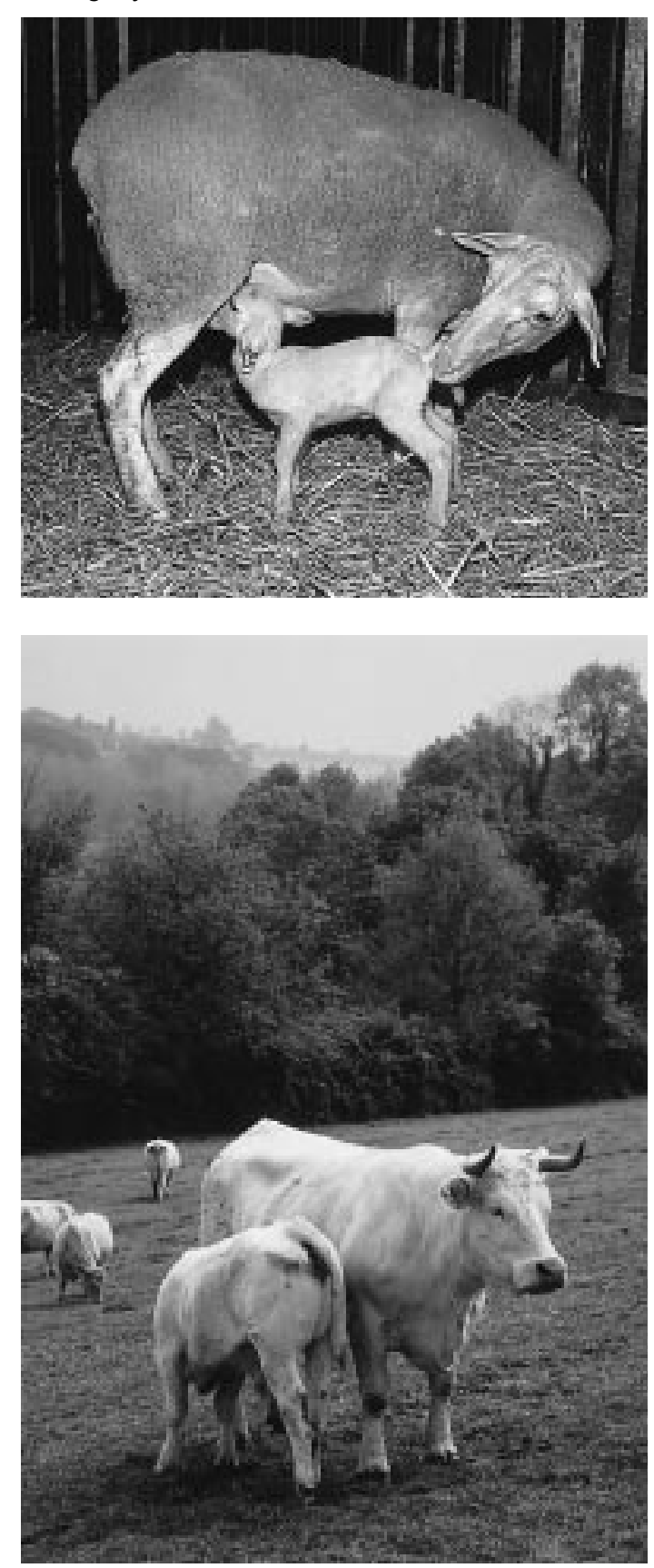

siologiques qui contrôlent l'expulsion du fœetus (figure 2). On peut en effet induire un comportement maternel chez des brebis non gestantes par un traitement combiné d'œstradiol et de progestérone qui mime les profils hormonaux de la fin de gestation (Poindron et al 1984). En fait, une injection d'œstradiol peut, à elle seule, permettre l'expression du comportement maternel. Cependant, l'action de l'œstradiol est rarement suffisante pour induire des soins similaires à ceux observés chez des femelles parturientes : la latence d'apparition du comportement maternel est souvent de plusieurs heures, les léchages sont absents ou apparaissent après l'acceptation à la mamelle. L'œstradiol préparerait en fait le système nerveux central à l'action de déclen- 
Figure 2. Existence d'un comportement maternel à différents stades d'un cycle de reproduction chez la brebis, en relation avec la concentration plasmatique d'œstradiol et de progestérone (Poindron et Le Neindre 1980).

$\%$ de brebis manifestant un comportement maternel
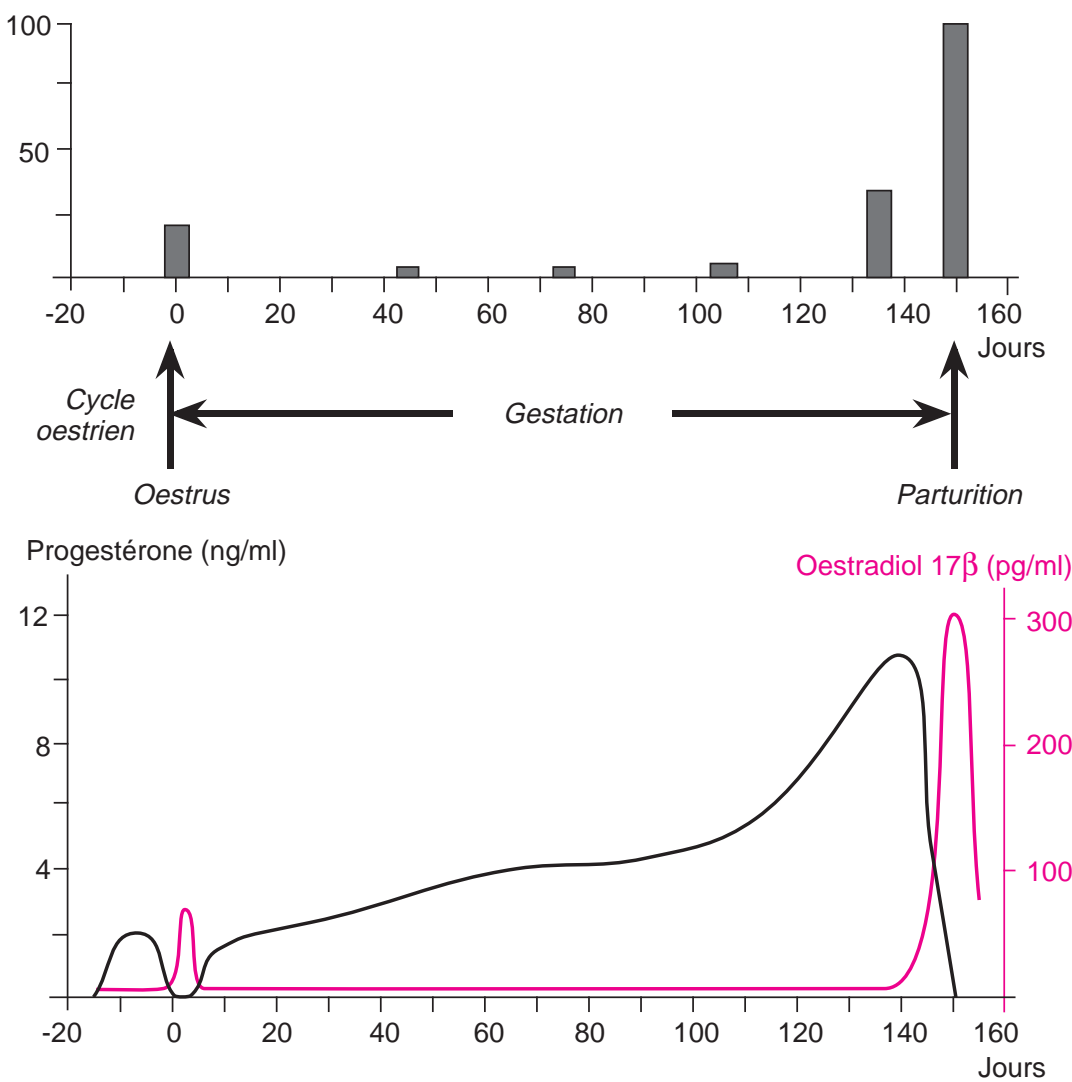

cheurs plus spécifiques. Ainsi, chez les ovins, c'est la stimulation vaginale causée par l'expulsion du fœtus qui assure l'apparition immédiate du comportement maternel dès la naissance du petit : une stimulation vaginale de 5 minutes chez des brebis non gestantes sous imprégnation œstrogénique, provoque l'apparition d'un comportement maternel complet dans les 5 à 10 minutes suivantes chez $80 \%$ des femelles, contre des latences allant jusqu'à 120 minutes et des taux de 60 $\%$ sans stimulation vaginale (Poindron et al 1984). Des résultats convergents ont été obtenus chez la chèvre (Romeyer et al 1994b). Cette stimulation cervico-vaginale agit au niveau du système nerveux central par l'intermédiaire d'au moins deux mécanismes. D'une part, elle est associée à la libération de noradrénaline, qui augmente pendant la parturition dans les ventricules cérébraux, ainsi que dans les bulbes olfactifs où elle régule la mémorisation de l'odeur du jeune. D'autre part, elle déclenche la libération centrale d'ocytocine qui facilite le comportement maternel chez la brebis et qui serait également impliquée dans la mise en en place du lien d'attachement (Poindron et al 1993).

\section{3 / Stratégies d'élevage du jeune}

La période néonatale est caractérisée par des interactions mère-jeune importantes chez tous les ruminants, hormis les camélidés (Lent 1974). Par la suite, les ruminants se subdivisent en deux groupes selon le type de relations spatiales qui se développent entre la mère et le jeune : les animaux de type "caché » et ceux de type "suivi » (traduction des termes anglais " hiders " et "followers ", Lent 1974). Chez les animaux de type "caché ", les relations mère-jeune sont caractérisées par de longues périodes de séparation. Peu après la naissance, le nouveau-né s'éloigne de sa mère et se dirige vers un lieu éloigné du site de mise-bas où il se cachera pour n'en sortir qu'au cours des périodes d'allaitement. C'est le cas du cerf, du chevreuil, des caprins et des bovins. Par contre, chez les animaux de type « suivi » le nouveau-né suit sa mère dans ses moindres déplacements et ceci dès les premières heures qui suivent la naissance. Il montre un comportement de détresse s'il est séparé de sa mère. Un tel comportement est observé chez le renne, le bison, les ovins et les camélidés.

Chez les espèces où le jeune reste caché, la mère peut s'en éloigner de plusieurs centaines de mètres voire plusieurs kilomètres. Mais la variabilité interindividuelle est importante et les conditions environnementales, notamment la qualité nutritionnelle du couvert végétal, influencent beaucoup les distances parcourues par la mère. La femelle ne revient alors que pour nourrir son petit, ce qui, pour certaines espèces, se traduit par deux à trois allaitements quotidiens. La mère se dirige rarement vers le lieu exact où se trouve son petit. Elle s'en approche mais c'est le jeune qui prend l'initiative de sortir de sa cachette et de se diriger vers sa mère. Cette réunion est généralement précédée par des échanges vocaux qui permettent l'identification du partenaire. Après la tétée, le jeune peut choisir un nouvel endroit pour se cacher, un comportement qui est interprété comme une stratégie d'évitement des prédateurs. La période durant laquelle le jeune reste caché varie selon les espèces, elle n'est que de quelques jours chez le cerf et les caprins, mais peut durer jusqu'à trois semaines chez le chevreuil (Alexander 1988). Par la suite, celui-ci intègre le groupe matriarcal.

Chez les espèces de type "suivi ", le nouveau-né, à peine debout, présente de fortes aptitudes à suivre tout objet en mouvement. On a pu montrer qu'en conditions extensives, un agneau âgé de 2 jours pouvait suivre sa mère sur 2 à 3 kilomètres sans difficultés. La mère et le jeune réagissent fortement à la séparation : ils émettent de nombreux cris de détresse s'ils sont séparés l'un de l'autre et ceci dès la naissance. Au cours des 3-4 jours qui suivent la parturition, mère et jeune ne s'éloignent guère de plus de quelques mètres. Ce n'est que par la suite que cette distance augmente, lorsque l'agneau rejoint des congénères de même âge pour former des groupes de jeu. Dans ce type de relation, les tétées sont extrêmement fréquentes. L'agneau tète environ toutes les heures au cours de la première semaine de vie, le lama trois fois plus. 
Une autre caractéristique de ces animaux est que le jeune présente très tôt des réactions de fuite : au moindre mouvement de panique de la mère, ou même d'un autre adulte, le jeune se met aussitôt à courir. Son rapide développement moteur et sa tendance à suivre un adulte en mouvement lui permettent de fuir tout prédateur potentiel. De plus, la synchronisation des parturitions diminue, par un effet du nombre, le taux de mortalité par prédation, et la présence de congénères autour de la femelle parturiente peut dissuader un prédateur d'attaquer, surtout si ceux-ci sont suceptibles d'agresser le prédateur. Aussi les éleveurs nord-américains procèdent-ils à des élevages mixtes afin d'utiliser le comportement agressif des bovins pour protéger les agneaux contre les attaques de chacals (Hulet et al 1987).

\section{4 / Reconnaissance interindividuelle}

C'est chez les ruminants domestiques que la plupart des études concernant la reconnaissance mère-jeune ont été entreprises (bovins : Le Neindre 1984, ovins et caprins : Poindron et al 1993). En ce qui concerne la mère, c'est surtout son comportement sélectif qui a été retenu comme preuve d'une reconnaissance : elle n'accepte d'allaiter que son propre jeune et exclut tout étranger. Toutefois, si la mise en place d'un comportement sélectif nécessite obligatoirement l'identification du partenaire, l'inverse n'est pas vrai. La sélectivité des femelles envers un jeune varie beaucoup selon leur type génétique : les vaches des races laitières tolèrent davantage un jeune étranger que les vaches des races rustiques (Le Neindre 1984). Cette tolérance à l'égard des étrangers n'exclut toutefois pas une recon- naissance mère-jeune : une mère peut très bien être capable d'identifier son jeune tout en acceptant d'allaiter un étranger. Chez le buffle, il semble que la femelle allaite spontanément des jeunes étrangers ; néanmoins l'allaitement de son jeune est beaucoup plus fréquemment observé (Murphey et al 1991).

Les études concernant la reconnaissance mutuelle mère-jeune ont été particulièrement nombreuses chez les ovins (figure 3). Elles ont montré que la reconnaissance à distance de l'agneau par sa mère reposait sur des signaux acoustiques et visuels alors que la reconnaissance proximale (moins de $25 \mathrm{~cm}$ ) et l'acceptation finale de l'agneau à la tétée s'appuyait essentiellement sur des critères olfactifs (Poindron et Le Neindre 1980). Il semble d'ailleurs que ce soit une généralité chez les ruminants. Cette reconnaissance olfactive du nouveau-né se met en place dans les 4 heures post-partum et la reconnaissance à distance est clairement établie dès $12 \mathrm{~h}$ (Ferreira 1998, communication personnelle). La perte de l'odorat (anosmie) entraîne une absence totale de sélectivité maternelle : la brebis allaite alors tout agneau étranger qui lui est présenté. Par contre, l'anosmie n'empêche pas la brebis d'identifier son agneau sur d'autres critères puisqu'à $12 \mathrm{~h}$ des brebis sans odorat reconnaissent leur agneau à distance aussi bien que des brebis intactes (Ferreira 1998, communication personnelle). L'agneau reconnaît également sa mère très tôt, entre 12 et $24 \mathrm{~h}$ après la naissance (Nowak et al 1990). Les signaux impliqués dans cette recherche active de la mère et son identification sont essentiellement acoustiques et visuels. Comme chez la brebis, l'absence d'odorat n'empêche pas l'agneau de reconnaître sa mère (Nowak 1991). Chez les bovins, la reconnaissance du veau par sa mère repose sur la conjonction de signaux olfactifs et visuels (Le Neindre 1984). Les veaux se basent davan-

\section{La mère reconnaît son jeune rapidement après la naissance, d'abord sur la base de signaux olfactifs, puis visuels et auditifs.}

Figure 3. Dynamique de reconnaissance mère-jeune chez les ovins. A gauche: pourcentage de brebis n'acceptant que leur agneau au cours d'un test où son jeune est remplacé par un agneau étranger de même âge (Poindron et Le Neindre 1980). A droite : pourcentage d'agneaux choisissant leur mère dans un parc de test où ils doivent la discriminer d'une brebis étrangère (Nowak et al 1990).
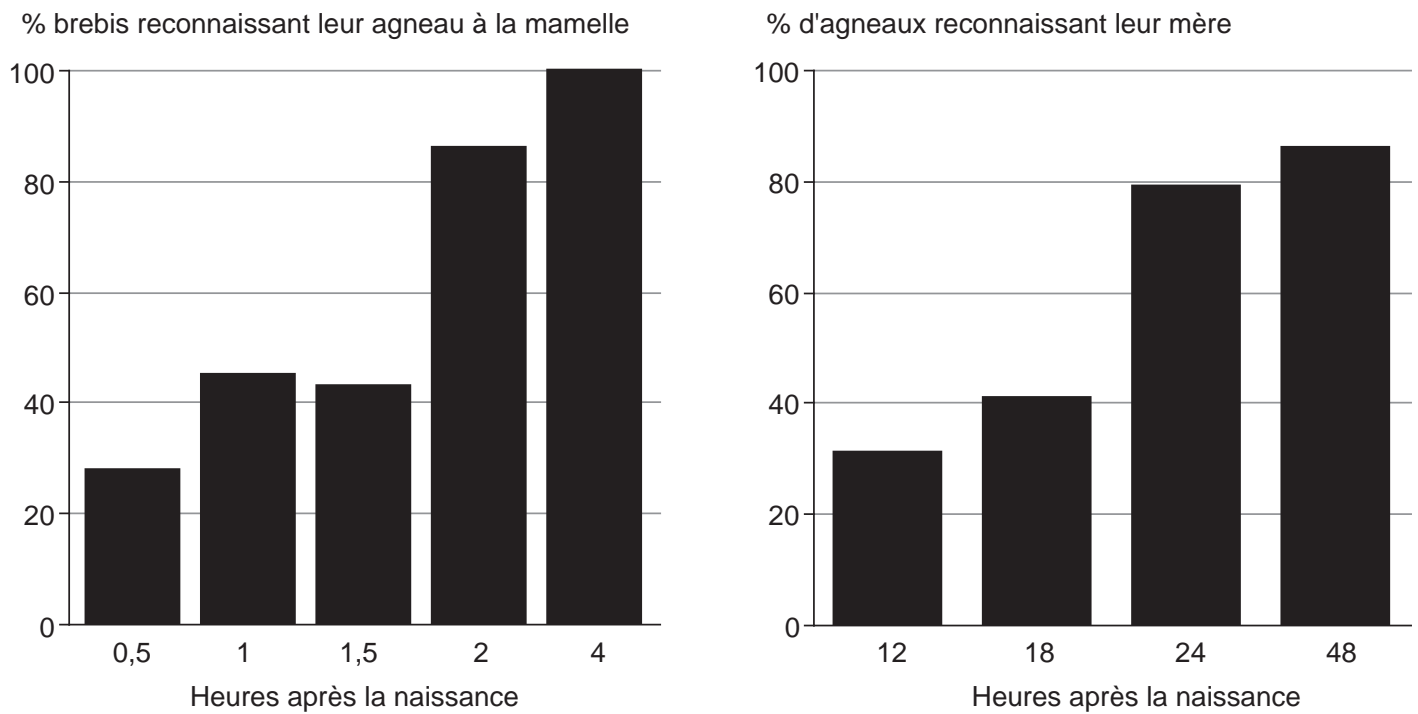
tage sur des critères visuels et auditifs. A l'âge d'un mois, ils discriminent les meuglements de leur mère par rapport à ceux d'une vache étrangère (Barfield et al 1994). A l'étable, lorsque les femelles sont silencieuses, ils utilisent la couleur du pelage de leur mère pour la retrouver parmi plusieurs vaches (Murphey et al 1990). Chez les caprins, la reconnaissance du chevreau par sa mère est sous contrôle olfactif dans les quelques heures qui suivent la parturition (Romeyer et al 1994a) avant qu'une reconnaissance acoustique ne se développe à partir du quatrième jour post-partum (Lenhardt 1977). Le chevreau peut également reconnaître sa mère dès l'âge de 2 jours (Lickliter et Heron 1984). Les signaux visuels, au moins à partir de l'âge de 5 jours, et probablement aussi les signaux olfactifs et auditifs doivent jouer un rôle primordial.

Chez les autres ruminants, le nombre d'études concernant la reconnaissance interindividuelle est relativement réduit. Chez le renne, mère et jeune sont capables de s'identifier l'un l'autre grâce aux signaux acoustiques et le jeune peut également être reconnu par ses caractéristiques olfactives (Espmark 1971a et 1971b, Källquist et Mossing 1982). Chez le cerf, il semble que la reconnaissance auditive soit mieux établie chez le jeune âgé de moins d'un mois que chez la mère (Vankova et al 1997). Toutefois, si la biche ne répond pas spécifiquement aux vocalisations de son faon, les études concernant les activités de tétée montrent clairement que la mère refuse d'allaiter les jeunes étrangers, suggérant que la biche se base davantage sur des critères olfactifs (Kelly et Drew 1976).

\section{5 / Relations mère-jeune et production animale}

\section{1 / Comportements mal adaptés pour la survie du nouveau-né}

La mortalité des jeunes se trouve invariablement concentrée dans les quelques jours qui suivent la naissance. Différents facteurs sont responsables de cette mortalité élevée, mais pour les espèces sauvages aussi bien que domestiques, la qualité des relations mèrejeune peut jouer un rôle important surtout en élevage extensif. Une tétée retardée à cause d'une femelle tant soit peu agitée, une absence de soins maternels et des nouveaunés abandonnés par leur mère sont autant de causes potentielles de mortalité. L'absence d'intérêt pour le nouveau-né à la parturition conduit presque inévitablement à sa mort lorsque l'agnelage n'est pas surveillé. L'agneau n'a alors que peu de chance d'être spontanément adopté par une autre brebis à moins que celle-ci soit sur le point de mettre bas. Le maintien du contact entre la brebis et sa portée revêt une importance particulière-
Certaines brebis abandonnent parfois une partie de leur portée. Cliché R. Nowak.

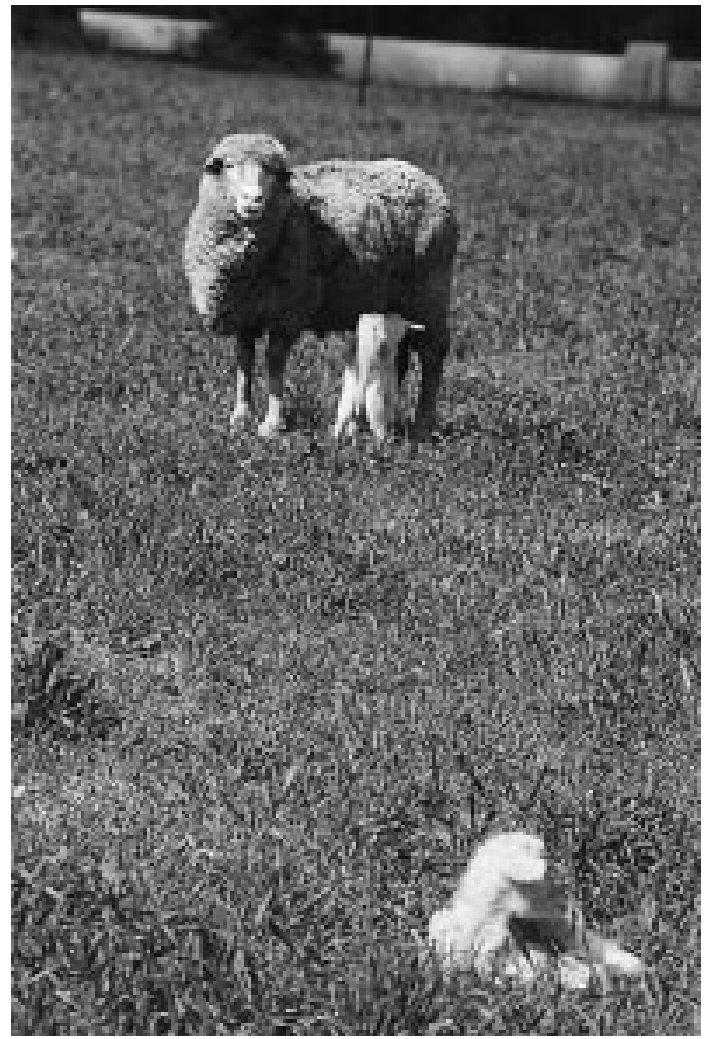

ment grande dans les élevages en plein air lorsque la prolificité est élevée (Stevens et al 1982, Lécrivain et Janeau 1988). Certaines mères semblent lentes à apprendre qu'elles ont plusieurs agneaux à élever et abandonnent parfois une partie de leur portée au cours des premières $24 \mathrm{~h}$, lorsqu'elles s'éloignent du lieu de mise-bas. Ceci est particulièrement vrai pour les brebis Mérinos australiennes (Stevens et al 1982) : la plus forte mortalité néonatale observée chez les jumeaux comparée à celle des agneaux simples pourrait être expliquée par ce phénomène. Stevens et al (1982) ont observé que 26 $\%$ des jumeaux nés de brebis multipares se trouvaient séparés définitivement de leur mère, ce qui conduit à une mort inévitable. Chez d'autres races, un effet de la parité est observé, les abandons étant plus fréquents chez les primipares que chez les multipares (Lécrivain et Janeau 1988). Toutefois, une participation active de l'agneau a été soulignée. D'une part, Stevens et al (1984) ont montré que le maintien du contact mère-jeune était aussi dû aux aptitudes des jumeaux à suivre leur mère dans ses déplacements. D'autre part, les agneaux jumeaux qui reconnaissent leur mère dès l'âge de $12 \mathrm{~h}$ ont de meilleures chances de survie ultérieure que ceux qui ne présentent pas ces aptitudes de reconnaissance (Nowak et Lindsay 1992). La qualité des liens mère-jeune est également influencée par le temps passé par la femelle parturiente sur le site de mise-bas : les abandons sont d'autant moins fréquents que les brebis y séjournent longtemps. Chez les Mérinos, il faut que la mère reste au moins $6 \mathrm{~h}$ sur 
le site de mise-bas avec ses jumeaux pour pallier tout risque d'abandon. Ces quelques heures sont nécessaires à la fois pour que la brebis établisse un lien d'attachement avec l'intégrité de sa portée et pour que les agneaux développent une motivation ou des aptitudes suffisantes pour suivre leur mère dans ses déplacements. Il convient donc, lorsqu'on veut augmenter la prolificité d'animaux élevés en plein air, de rechercher une race dont les mères restent longtemps sur le site de mise-bas et de sélectionner de tels individus au sein d'une même race. Par ailleurs, on a pu montrer que l'expression du comportement maternel était relié au niveau d'émotivité de la brebis. Certains critères d'émotivité sont héritables et peuvent être pris en compte dans des programmes de sélection génétique. C'est ainsi que O'Connor et al (1985) ont pu améliorer le rapport nombre d'agneaux sevrés / nombre d'agneaux nés en sélectionnant les brebis sur leur réaction de peur vis-à-vis de l'homme : ce rapport était d'autant plus élevé que les brebis étaient moins peureuses, en particulier chez celles allaitant des agneaux multiples. Enfin, les relations mère-jeune peuvent être également modulées par le niveau de nutrition pendant la gestation. Les effets néfastes d'une sous-nutrition sévère ont été rapportés chez le mouton (Thomson et Thomson 1949, Putu et al 1988) et le cerf de virginie (Langenau et Lerg 1976 ; tableau 1). Cela se traduit par une absence de comportement maternel chez les femelles sous-alimentées et une morti-natalité accrue de leurs jeunes. A l'inverse, un supplément alimentaire à base de graines de lupin améliore sensiblement le comportement de la mère et la survie des agneaux, surtout chez les jumeaux (Putu et al 1988). On ne sait encore expliquer cette action de l'alimentation sur le comportement maternel. L'état corporel des femelles sous-alimentées peut en être la cause puisque, étant plus faiblement constituées, elles sont davantage épuisées par les efforts déployés à la parturition. Toutefois, un effet direct de certains nutriments ou de la fonction digestive sur le système nerveux central, et donc sur l'expression du comportement maternel, est aussi envisageable.

\section{2 / L'adoption : difficilement réalisable chez les ruminants}

En raison de son déterminisme physiologique, le comportement maternel peut apparaître dans les heures et même parfois les jours qui précèdent la parturition. Il s'agit là d'un phénomène normal. L'inconvénient réside dans le fait que ceci peut conduire à l'adoption temporaire ou définitive d'un nouveau-né étranger. Le phénomène sera d'autant plus fréquent que le nombre de femelles approchant du terme et leur densité sont élevées (ovins : Alexander et al 1983). Il est accentué chez les races prolifiques et les conséquences de ces adoptions sont variables. Si l'adoption a lieu plusieurs jours avant que la brebis adoptive ne mette bas, l'agneau peut mourir de sous-alimentation. Aux alentours de la parturition, l'adoption peut être permanente, c'est le cas le plus fréquent. La brebis adoptive peut élever deux ou trois agneaux, alors qu'une autre femelle sera tarie : il y a

Brebis pré-parturiente léchant les agneaux d'une brebis venant juste d'agneler. Cliché P. Poindron.

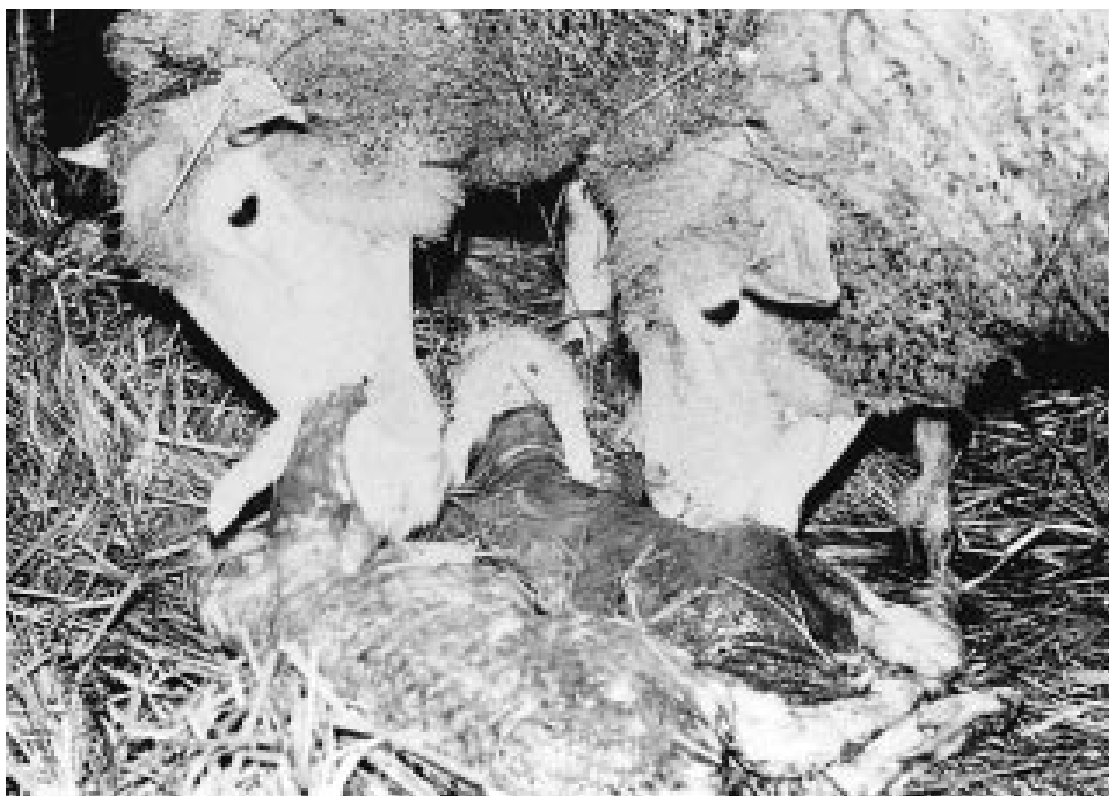

Tableau 1. Relation entre le niveau alimentaire pendant la gestation (+ : alimentation ad libitum ; - : alimentation inférieure aux besoins), l'expression du comportement maternel à la parturition et la mortalité néonatale liée au comportement inadapté des mères.

\begin{tabular}{|c|c|c|c|c|c|c|c|}
\hline \multirow[b]{3}{*}{ Nutrition } & \multirow{3}{*}{$\begin{array}{c}\text { Taille } \\
\text { de la portée }\end{array}$} & \multicolumn{4}{|c|}{$\begin{array}{l}\% \text { de femelles } \\
\text { ayant un comportement maternel }\end{array}$} & \multirow{2}{*}{\multicolumn{2}{|c|}{$\begin{array}{c}\text { \% mortalité } \\
\text { néonatale } \\
(0-48 \mathrm{~h})\end{array}$}} \\
\hline & & \multicolumn{2}{|c|}{ normal } & \multicolumn{2}{|c|}{$\begin{array}{c}\text { anormal } \\
\text { (rejet, abandon, } \\
\text { agression) }\end{array}$} & & \\
\hline & & + & - & + & - & + & - \\
\hline Brebis Mérinos & Simples & 87 & 71 & 13 & 29 & 2 & 6 \\
\hline (Putu et al 1988) & Jumeaux & 87 & 50 & 13 & 50 & 15 & 33 \\
\hline Cerf de Virginie & Simples & 100 & 88 & 0 & 12 & 0 & 3 \\
\hline (Langeneau et Lerg 1976) & Jumeaux & 97 & 83 & 3 & 17 & 0 & 15 \\
\hline
\end{tabular}


Figure 4. Efficacité de différentes techniques utilisées pour l'adoption d'un agneau étranger par une brebis dont l'agneau est mort peu après la naissance. Les différents traitements sont appliqués pendant quelques heures sur l'agneau mort, puis celui-ci est remplacé par l'agneau étranger (Alexander et al 1987).

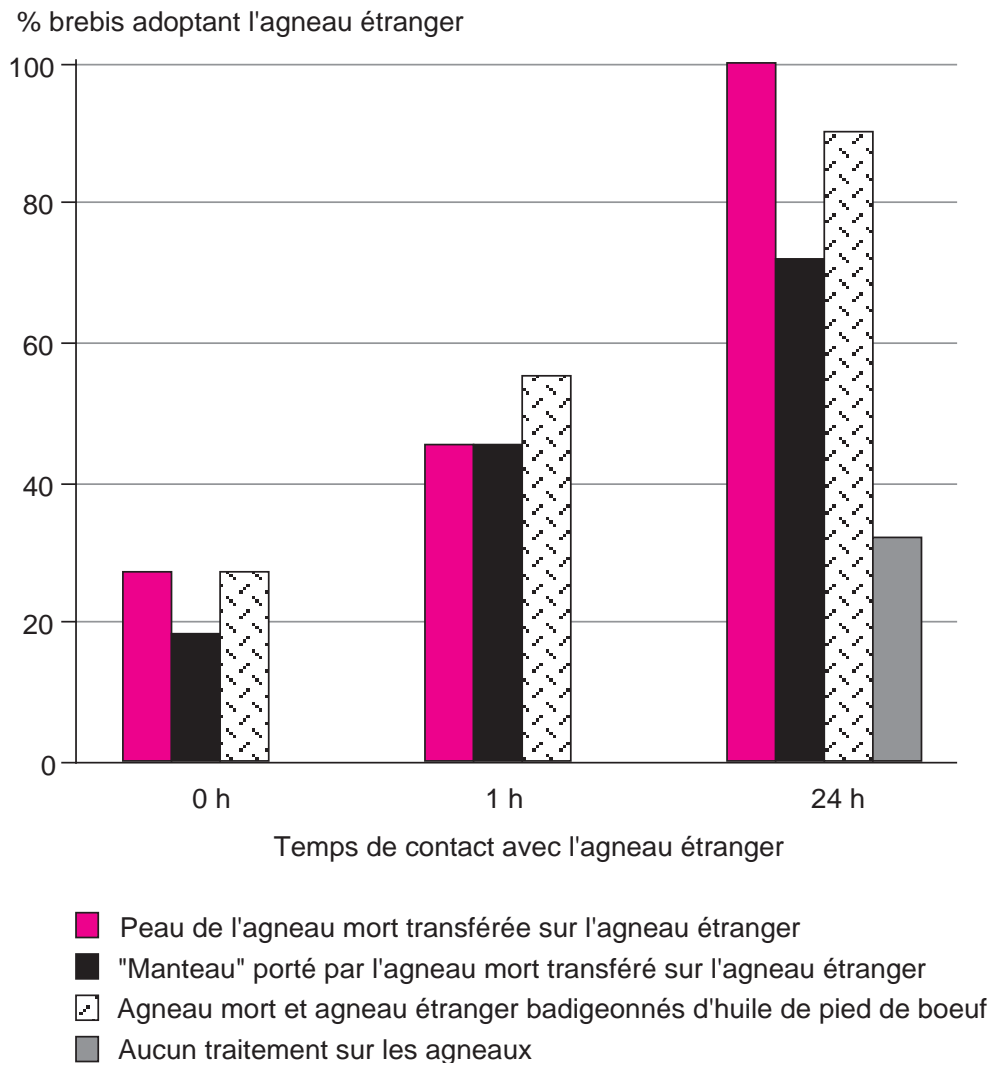

Figure 5. Efficacité de différentes techniques utilisées pour l'adoption d'un agneau par une brebis qui en allaite déjà un. Les différents traitements sont appliqués 1 à 2 jours après la parturition et les tests d'adoption commencent le lendemain (Alexander et Stevens 1985).

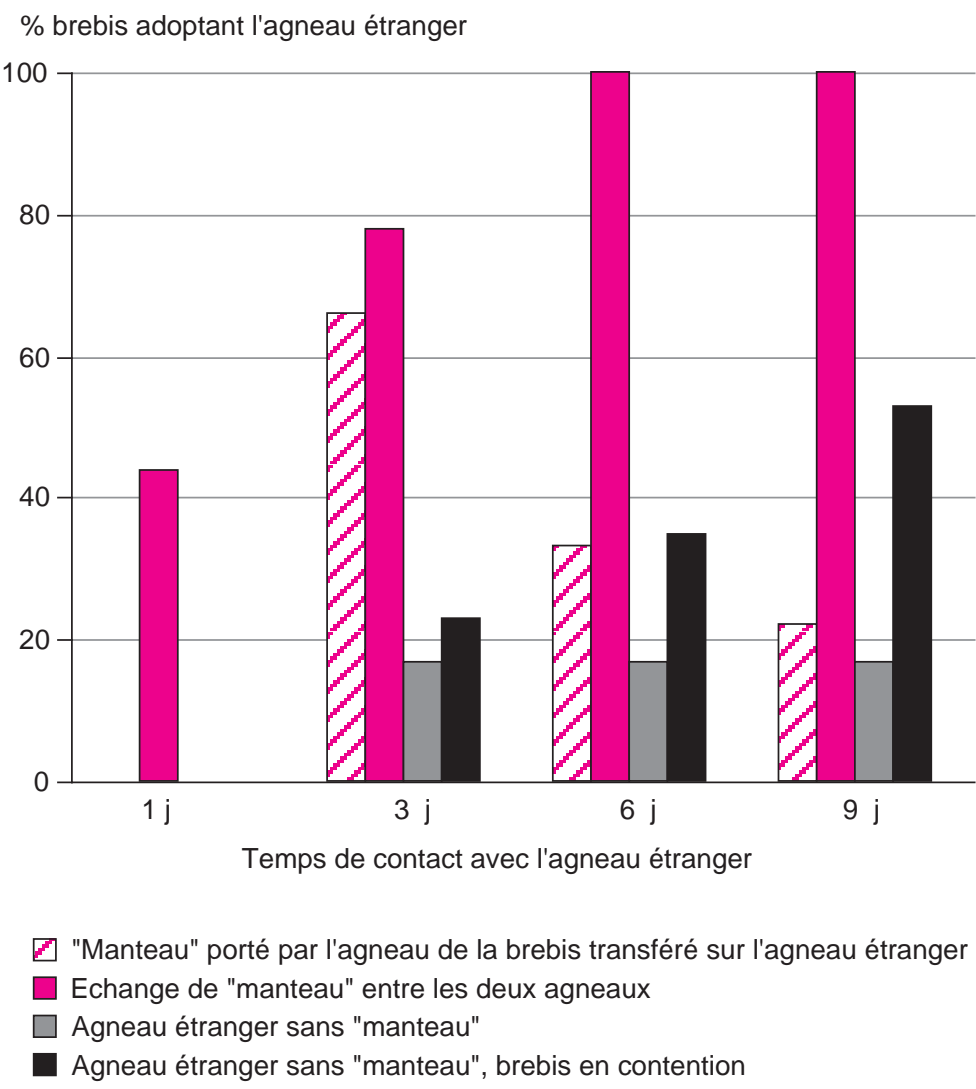

alors mauvaise utilisation du potentiel laitier. Si les adoptions spontanées sont extrêmement rares chez les ruminants en dehors de la période péri-partum, il semble par contre que les buffles fassent exception. En effet Murphey et al (1991) signalent que les femelles non seulement se laissent téter par des jeunes étrangers mais vont aussi jusqu'à adopter des orphelins.

Que quelques femelles adoptent spontanément un jeune étranger est une chose. Vouloir réaliser l'adoption systématique d'un jeune chez un grand nombre d'animaux en est une autre. L'adoption spontanée n'étant possible qu'autour de la mise-bas, il faut recourir à tout autre moment à des techniques plus ou moins élaborées (ovins : Alexander et Stevens 1985, Alexander et al 1987 et 1989, bovins : Le Neindre et Garel 1979). Qu'il s'agisse de substituer un jeune à un autre (cas de femelles ayant perdu leur petit) ou d'ajouter un jeune à une femelle en ayant déjà un, le problème de base est le même : il faut transférer les caractéristiques olfactives de l'agneau familier vers l'agneau à adopter ou masquer celles de l'agneau étranger jusqu'à son acceptation définitive par la brebis. Dans le premier cas, la méthode la plus connue consiste à recouvrir le jeune étranger avec la peau de celui qui est mort afin de faciliter son acceptation. D'autres méthodes sont préconisées, mais dans tous les cas le traitement doit d'abord être appliqué à l'agneau mort qui sera laissé avec sa mère pendant quelques heures, avant de l'appliquer à l'étranger qui sera présenté après retrait de l'agneau mort. C'est ainsi qu'un manteau de toile de jute porté par l'agneau mort mis sur l'agneau à adopter peut se montrer efficace, de même que le badigeonnage des corps des agneaux avec de l'huile de pied de bœuf (figure 4). Les techniques utilisées pour faire adopter un agneau à une brebis qui en allaite déjà un sont le plus souvent l'utilisation de masquants odorants, le transfert de l'odeur du jeune familier vers l'étranger, et utilisation de tranquillisants sur la femelle. Dans ce cas, les meilleurs traitements consistent à masquer l'odeur de l'agneau étranger ou à mettre la brebis en contention (figure 5). Quelle que soit la méthode utilisée, la mère et le jeune doivent être temporairement isolés du troupeau et mis dans un espace restreint afin de forcer le contact. L'efficacité des méthodes disponibles est très variable et dépend beaucoup du savoir-faire de l'éleveur.

\section{Conclusions}

Chez les ruminants, l'établissement du lien mère-jeune nécessite un comportement adapté de la part de la mère et de son nouveau-né. Plusieurs facteurs sont impliqués dans la réalisation de ce processus d'attachement : (i) l'apparition soudaine de l'intérêt pour le nouveau-né au moment de la parturition, (ii) l'établissement en quelques heures du lien sélectif avec le jeune, (iii) une activité 
suffisante du nouveau-né pour lui permettre de localiser rapidement la mamelle, d'apprendre à reconnaître sa mère et de la suivre dans ses déplacements (chez les « suivis»), ou de se cacher à l'abri des prédateurs (chez les « cachés »). L'importance du comportement maternel en production animale varie en fonction de l'espèce et du type d'élevage. D'une manière générale, le comportement maternel, de par sa sélectivité, est un frein à la réalisation d'adoptions, sauf au moment de la partu- rition. Quant à l'absence de comportement maternel chez les femelles primipares, ou l'abandon d'un jeune chez les femelles de portées multiples, ils peuvent être des causes non négligeables de mortalité en conditions extensives.

Une première version de ce texte a été publiée au cours des $3^{\text {es }}$ Rencontres autour des Recherches sur les Ruminants (Paris, les 4 et 5 décembre 1996).

\section{Références bibliographiques}

Agabriel J., Bony J., Micol D., 1996. Elevage du bison d'Amérique (Bison Bison). INRA Prod. Anim., 9, 379-388.

Alexander G., 1988. What makes a good mother ? Components and comparative aspects of maternal behaviour in ungulates. Proc. Aust. Soc. Anim. Prod., $17,25-41$

Alexander G., Stevens D., 1985. Fostering in sheep. II. Use of hessian coats to foster additionnal lambs onto ewes with single lambs. Appl. Anim. Behav. Sci., 14, 335-344.

Alexander G., Stevens D., Mottershead B., 1983. Problems in the accurate recording of lambing data. Aust. J. Exp. Agric. Husb., 23, 361-368.

Alexander G., Stevens D., Bradley L.R., 1987. Fostering in sheep : fostering lambs onto ewes whose lambs have died soon after birth. Aust. J. Exp. Agric., 27, 765-769.

Alexander G., Stevens D., Bradley L.R., 1989. Fostering in sheep : an exploratory comparison of several approaches. Aust. J. Exp. Agric., 29, 509-512.

Arman P., 1974. A note on parturition and maternal behaviour in captive red deer (Cervus elaphus L.). J. Reprod. Fert., 37, 87-90.

Barfield C.H., Tang-Martinez Z., Trainer J.M., 1994. Domestic calves (Bos taurus) recognize their own mothers by auditory cues. Ethology, 97, 257-264.

Espmark Y., 1971a. Mother-young relationship and ontogeny of behaviour in reindeer (Rangifer tarandus L.). Z. Tierpsychol., 29, 42-81.

Espmark Y., 1971b. Individual recognition by voice in reindeer mother-young relationship. Field observations and playback experiments. Behaviour, 40, 295-301.

Faye B., Jouany J.P., Chacornac J.P., Ratovonanahary M., 1995. L'élevage des grands camélidés. Analyse des initiatives réalisées en France. INRA Prod. Anim., 8, 3-17.

Gauthier-Pilters H., Dagg A.I., 1981. The camel : its evolution, ecology, behavior and relationship to man. University of Chicago Press, Chicago.

Hulet C.V., Anderson D.M., Smith J.N., Shupe W.L., 1987. Bonding of sheep to cattle as an effective technique for predation control. Appl. Anim. Behav. Sci., $19,19-25$.

Källquist L., Mossing T., 1982. Olfactory recognition between mother and calf in reindeer (Rangifer $T$. Tarandus L.). Appl. Anim. Ethol., 8, 561-565.
Kelly R.W., Drew K.R., 1976. Shelter seeking and sucking behaviour of the red deer calf (Cervus elaphus) in a farmed situation. Appl. Anim. Ethol., 2, 101-111.

Langenau E.E., Lerg J.M., 1976. The effects of winter nutritional stress on maternal and neonatal behavior in penned white-tailed deer. Appl. Anim. Ethol., 2, 207-223.

Lécrivain E., Janeau G., 1988. Mortalité néonatale d'agneaux nés en plein air sans aide de l'éleveur. INRA Prod. Anim., 1, 331-338.

Le Neindre P., 1984. La relation mère-jeune chez les ovins : influence de l'environnement social et de la race. Thèse de doctorat d'état, Université de Rennes.

Le Neindre P., Garel J.P., 1979. Adoption d'un deuxième veau par des vaches plusieurs jours après la mise-bas. Ann. Zootech., 28, 231-234.

Lenhardt M.L., 1977. Vocal contour cues in maternal recognition of goat kids. Appl. Anim. Ethol., 3, 211219 .

Lent P.C., 1974. In : V. Geist et F. Walther (eds), Behaviour of ungulates and its relation to management, 14-55. International Union for the Conservation of Nature and Natural Resources, Morges, Suisse.

Lévy F., 1985. Contribution à l'analyse des mécanismes de mise en place du comportement maternel chez la brebis (Ovis aries L.) : étude de la répulsion et de l'attraction vis-à-vis du liquide amniotique, mise en évidence, déterminisme, rôle. Thèse de doctorat d'état, Université de Paris VI.

Lévy F., Poindron P., Le Neindre P., 1983. Attraction and repulsion by amniotic fluids and their olfactory control in the ewe around parturition. Physiol. Behav., 31, 687-692.

Lickliter R.E., Heron J.R., 1984. Recognition of mother by newborn goats. Appl. Anim. Behav. Sci., $12,187-192$.

Murphey R.M., Ruiz-Miranda C.R., Duarte F.A.M., 1990. Maternal recognition in Gyr (Bos indicus) calves. Appl. Anim. Behav. Sci., 27, 183-191.

Murphey R.M., Paranhos da Costa M., de Souza Lima L.O., de Moura Duarte F.A., 1991. Communal suckling in water buffalo (Bubalus bubalis). Appl. Anim. Behav. Sci., 28, 341-352.

Nowak R., 1991. Senses involved in discrimination of merino ewes at close contact and from a distance by their newborn lambs. Anim. Behav., 42, 357-366. 
Nowak R., 1996. Neonatal survival : contributions from behavioural studies in sheep. Appl. Anim. Behav. Sci., 49, 61-72.

Nowak R., Lindsay D.R., 1992. Discrimination of merino ewes by their newborn lambs : important for survival ? Appl. Anim. Behav. Sci., 34, 61-74.

Nowak R., Poindron P., Putu I.G., 1990. Development of mother discrimination by single and multiple newborn lambs. Dev. Psychobiol., 22, 833-845.

O'Connor C.E., Jat N.P., Nico A.M., Beatson P.R., 1985. Ewe maternal behaviour score and lamb survival. Proc. N.Z. Soc. Anim. Prod., 45, 159-162.

Poindron P., Le Neindre P., 1980. Endocrine and sensory regulation of maternal behavior in the ewe. Adv. Study Behav., 11, 75-119.

Poindron P., Le Neindre P., Lévy F., Keverne E.B., 1984. Les mécanismes de l'acceptation du nouveauné chez la brebis. Biol. Behav., 9, 65-88.

Poindron, P., Nowak, R., Lévy, F., Porter, R.H., Schaal B., 1993. Development of exclusive motheryoung bonding in sheep and goats. Oxford Rev. Reprod. Biol., 15, 311-364.

Prud'hon M., Cordesse R., de Rouville S., Thimonier J., 1993. Les camélidés sud-américains : le point des connaissances. INRA Prod. Anim., 6, 5-15.

Putu I.G., Poindron P., Lindsay D.R., 1988. A high level of nutrition during late pregnancy improves subsequent maternel behaviour of merino ewes. Proc. Aust. Soc. Anim. Prod., 17, 294-297.

Romeyer A., Poindron P., Orgeur P., 1994a. Olfaction mediates the establishment of selective bonding in goats. Physiol. Behav., 56, 693-700.

Romeyer A., Poindron P., Porter R.H., Lévy F., Orgeur P., 1994b. Establishment of maternal bonding and its mediation by vaginal stimulation in goats. Physiol. Behav., 55, 395-400.

Stevens D., Alexander G., Lynch J.J., 1982. Lamb mortality due to inadequate care of twins by merino ewes. Appl. Anim. Ethol., 8, 243-252.

Stevens D., Alexander G., Mottershead B., Lynch J.J., 1984. Role of the lambs in post-partum separation of ewes from twin lambs. Proc. Aust. Soc. Anim. Prod., 15, 751.

Thimonier J., Sempéré, A., 1989. La reproduction chez les cervidés. INRA Prod. Anim., 2, 5-21.

Thomson A.M., Thomson W., 1949. Lambing in relation to the diet of the pregnant ewe. British J. Nutr., $2,290-305$.

Vankova D., Bartos L., Malek J., 1997. The role of vocalization in the communication between red deer hinds and calves. Ethology, 103, 795-808.

Vince M.A., 1993. Newborn lambs and their dams : the interaction that leads to sucking. Adv. Study Behav., 22, 239-268.

\begin{abstract}
Mother-young relationship during the perinatal period in ruminants.

Between ruminant species a number similarities in maternal behaviour become evident immediately after parturition : consumption of foetal membranes and fluids (excepting camelidae), an interest in the neonate and care directed towards it. Maternal care focuses the new-born's attention towards the mother's body and favours the success of the first sucking bouts. After this initial phase lasting a few hours, ruminants can be divided into two categories according to the type of spatial association of mother and offspring : species that hide their young for a variable amount
\end{abstract}

Abstract of time (e.g. red deer, roe deer, goat, cattle) and those where the young follows the mother soon after birth (e.g. reindeer, sheep). The motheryoung relationship is also characterised by a process of inter-individual recognition that is well developed. Generally the establishment of the mother-young relationship takes place without major problems. However, under certain conditions, by its absence or its selectivity, maternal behaviour can become a factor limiting production.

Nowak R., 1998. Développement de la relation mèrejeune chez les Ruminants. INRA Prod. Anim., 11, 115-124. 